\title{
Astragalus membranaceus ameliorates renal interstitial fibrosis by inhibiting tubular epithelial-mesenchymal transition in vivo and in vitro
}

\author{
GUANG SHAN, XIANG-JUN ZHOU, YUE XIA and HUI-JUN QIAN \\ Department of Urology, Renmin Hospital of Wuhan University, Wuhan, Hubei 430060, P.R. China
}

Received November 14, 2014; Accepted January 29, 2016

DOI: $10.3892 /$ etm.2016.3152

\begin{abstract}
Epithelial-mesenchymal transition (EMT) induces the progression of renal tubulointerstitial fibrosis. Astragalus membranaceus (AM) is a traditional Chinese herbal medicine that has been demonstrated to exert anti-inflammatory and anti-cancer effects, in addition to protecting and supporting the immune system. The present study investigated the effects of AM on renal fibrosis. A mouse model of unilateral ureteral obstruction (UUO) was established and treated with various concentrations of AM (100, 200 or $400 \mathrm{mg} / \mathrm{kg} /$ day). Interstitial fibrosis markedly increased in the UUO mice. AM significantly reduced the obstruction-induced upregulation of $\alpha$-smooth muscle actin ( $\alpha$-SMA) and downregulation of E-cadherin in the kidneys of the UUO mice $(\mathrm{P}<0.05)$. Furthermore, AM treatment significantly inhibited the induction of EMT and the deposition of extracellular matrix. In addition, a transforming growth factor (TGF)- $\beta 1$-stimulated murine renal proximal tubule cell line (NRK-52E) was treated with various concentrations of AM $(10,20$, and $40 \mu \mathrm{g} / \mathrm{ml})$. E-cadherin expression levels significantly decreased and those of $\alpha$-SMA significantly increased in NRK-52E cells stimulated with TGF- $\beta 1$ in vitro $(\mathrm{P}<0.05)$. Co-treatment with AM reversed these effects $(\mathrm{P}<0.05)$, and AM treatment reduced TGF- $\beta 1$-induced expression and Smad2/3 phosphorylation $(\mathrm{P}<0.05)$. These results suggested that AM antagonizes tubular EMT by inhibiting the Smad signaling pathway.
\end{abstract}

\section{Introduction}

Renal interstitial fibrosis is the result of end-stage acute and chronic kidney diseases (1). Renal interstitial fibrosis is characterized by the activation of interstitial fibroblasts and the accumulation of extracellular matrix (ECM) components,

Correspondence to: Mr. Guang Shan, Department of Urology, Renmin Hospital of Wuhan University, 99 ZhangZhiDong Street, Wuhan, Hubei 430060, P.R. China

E-mail: shanguang1980@163.com

Key words: Astragalus membranaceus, epithelial-mesenchymal transition, renal fibrosis including fibronectin (FN) and collagen (2). Renal interstitial fibroblasts produce ECM during fibrosis, and ECM is also produced by epithelial cells, endothelial cells, pericytes, and bone marrow stem cells (3). Epithelial-mesenchymal transition (EMT) is an important signaling pathway in the progression of renal interstitial fibrosis (4), which represents the loss of epithelial cells and their adhesion molecules, including E-cadherin, and an increase in mesenchymal cells and markers, such as $\alpha$-smooth muscle actin (SMA) (5). Transforming growth factor (TGF)- $\beta 1$ is an important mediator that promotes EMT via myofibroblast development by inducing the expression of $\alpha$-SMA via various cytokines (6). The downstream Smad signaling pathway is activated by TGF- $\beta 1$ during renal fibrosis, which induces renal scarring (7). Therefore, manipulating downstream TGF- $\beta 1$ signaling represents a potent therapeutic target for the reversal of EMT and renal interstitial fibrosis.

Astragalus membranaceus (AM) is a traditional Chinese herbal medicine which is used to treat various ailments, including common colds, fatigue, anorexia, and cardiac diseases $(8,9)$. Previous studies have demonstrated the anti-fibrotic effects of AM on pulmonary fibrosis (10), liver fibrosis (11), and nephropathy (12) by inhibiting the activation of the TGF- $\beta 1$ signaling pathway. In the present study, the effects of AM on renal tubular EMT were examined in NRK-52E cells and a mouse model of unilateral ureteral obstruction (UUO) in order to elucidate whether AM can ameliorate renal fibrosis via the inhibition of EMT.

\section{Materials and methods}

Animals and experimental design. A total of 30 male C57BL6 mice (age, 8 weeks; weight, 18-20 g) were purchased from Wuhan University (Wuhan, China) and housed at the Experimental Animal Center of Wuhan University. The mice were maintained at $25^{\circ} \mathrm{C}$ under a 12 -h light/dark cycle, with ad libitum access to food and water. The purified natural product AM was obtained from Shanghai Yuanye Biotechnology Co., Ltd. (Shanghai, China). In order to establish a murine model of UUO, the mice were anesthetized with $1.5 \%$ sodium pentobarbital $(50 \mathrm{mg} / \mathrm{kg}$; Shanghai Haling Biological Technology Co., Ltd., Shanghai, China) and a left lateral incision was made on the back of the mice to expose the kidney. The left ureter was ligated with 4-0 silk sutures 
at the junction between the renal pelvis and ureter and the ligature position was consistent. Following completion, the incision was closed in layers. Sham-group mice received the same incision and closure procedures without ligation. UUO mice were treated by intraperitoneal injection of AM (100, 200 , and $400 \mathrm{mg} / \mathrm{kg} / \mathrm{day}$ ) ( $\mathrm{n}=6$ per group). Mice in the sham were administered equal volumes of sterile saline $(n=6$ per group). Mice were sacrificed by cervical dislocation following anesthetization on day 7 post-surgery, and the obstructed kidneys were harvested. Kidney samples were fixed in $4 \%$ buffered paraformaldehyde (Guge Biotechnology Co., Ltd., Wuhan, China) and were subsequently embedded in paraffin (Guge Biotechnology Co., Ltd.) for histological and immunohistochemical examination. The remaining kidneys were snap-frozen in liquid nitrogen and stored at $-80^{\circ} \mathrm{C}$ for protein extraction.

Cell culture and experimental design. NRK-52E cells were obtained from Boster Bioengineering Co., Ltd. (Wuhan, China) and cultured in Dulbecco's modified Eagle's medium supplemented with $10 \%$ fetal bovine serum (GE Healthcare Life Sciences, Logan, UT, USA) at $37^{\circ} \mathrm{C}$ in a humidified atmosphere containing $5 \% \mathrm{CO}_{2}$. A total of $1.5 \times 10^{6}$ cells $/ \mathrm{ml}$ cells were transferred to 6 -well plates and were divided into the following five treatment groups: Control; $5 \mathrm{ng} / \mathrm{ml}$ TGF- $\beta 1$ (R\&D Systems, Minneapolis, MN, USA); $5 \mathrm{ng} / \mathrm{mlTGF}-\beta 1+10 \mu \mathrm{g} / \mathrm{mlAM} ; 5 \mathrm{ng} / \mathrm{mlTGF}-\beta 1+20 \mu \mathrm{g} / \mathrm{mlAM}$; and $5 \mathrm{ng} / \mathrm{ml} \mathrm{TGF-} \beta 1+40 \mu \mathrm{g} / \mathrm{ml} \mathrm{AM}$.

Immunohistochemistry staining. Kidney sections (5- $\mu \mathrm{m})$ were prepared using a microtome (RM3225; Leica Geosystems, Milton Keynes, UK). The tissue sections were placed on slides, deparaffinized in xylene (Boster Bioengineering Co., Ltd.) and hydrated in graded ethanol prior to treatment with $3 \%$ hydrogen peroxide solution (Guge Biotechnology Co., Ltd.) for $15 \mathrm{~min}$ at room temperature. Following three washes with phosphate-buffered saline (PBS), the antigens were retrieved by boiling the tissue sections in Tris-ethylenediaminetetraacetic acid (EDTA) buffer (12.1 g Tris base; 3.7 g EDTA; $500 \mathrm{ml} \mathrm{H}_{2} \mathrm{O}$; Boster Bioengineering Co., Ltd.) for $15 \mathrm{~min}$ in a microwave oven on high power. Following cooling, the tissue sections were washed three times with PBS and incubated with the following primary antibodies at $37^{\circ} \mathrm{C}$ for $1 \mathrm{~h}$ : Mouse anti- $\alpha$-SMA monoclonal antibody (1:100; BM0002; Boster Bioengineering Co., Ltd.), mouse anti-E-cadherin monoclonal antibody (1:50; 610181; BD Biosciences, Franklin Lakes, NJ, USA), rabbit anti-collagen I polyclonal antibody (1:200; BA0326; Boster Bioengineering Co., Ltd.), and rabbit anti-FN polyclonal antibody (1:200; ab2413; Abcam, Cambridge, UK). Subsequently, the tissue sections were washed three times with PBS and incubated with horseradish peroxidase (HRP)-conjugated goat anti-rabbit/mouse polyclonal secondary antibody (1:100; BA1003; Boster Bioengineering Co., Ltd.) for $10 \mathrm{~min}$ at $37^{\circ} \mathrm{C}$. The sections were treated with 3,3'-diaminobenzidine (Beyotime Institute of Biotechnology, Shanghai, China) to develop a color reaction, and the reaction was subsequently terminated by washing twice with distilled water. The tissue sections were finally counterstained with hematoxylin (Guge Biotechnology Co., Ltd.).
Immunofluorescence staining. Cells were fixed in $4 \%$ paraformaldehyde for $30 \mathrm{~min}$ at room temperature and subsequently permeabilized with $0.1 \%$ Triton X-100 (Boster Bioengineering Co., Ltd.) in PBS for 5 min prior to rinsing three times with PBS for 5 min. Following blocking in 5\% bovine serum albumin (BSA; Guge Biotechnology Co., Ltd.) and $1 \%$ Triton $\mathrm{X}-100 / \mathrm{PBS}$ for $30 \mathrm{~min}$ at room temperature, the cells were incubated with primary antibodies targeting $\alpha$-SMA (1:100), E-cadherin (1:50) and FN (1:200) overnight at $4^{\circ} \mathrm{C}$. The cells were then cultured with cyanine 3-goat anti-mouse immunoglobulin G (IgG; 1:100; GB21303) and fluorescein isothiocyanate-goat anti-rabbit IgG (1:100; GB22303; both Boster Bioengineering Co., Ltd.) for $1 \mathrm{~h}$. The cell nuclei were stained with 4'6'-diamino-2-phenylindole dihydrochloride (Beyotime Institute of Biotechnology).

Reverse transcription-quantitative polymerase chain reaction $(R T-q P C R)$. Total RNA was isolated from renal cortex samples and NRK-52E cells using TRIzol ${ }^{\circledR}$ reagent (Invitrogen; Thermo Fisher Scientific, Inc., Waltham, MA, USA), according to the manufacturer's protocol. RNA underwent purification with DNase (Qiagen, Inc., Valencia, CA, USA), after which the concentration of RNA was measured using a spectrophotometer (Nanodrop ${ }^{\mathrm{TM}} 2000$; Thermo Fisher Scientific, Inc.) at $260 \mathrm{~nm}$, and the purity was assessed by determining the absorbance ratio at A260/A280. Subsequently, RNA $(20 \mu \mathrm{l})$ was reverse transcribed into cDNA using the RevertAid First Strand cDNA Synthesis kit (cat. no. K1622; Thermo Fisher Scientific, Inc.). qPCR was performed using the iQ SYBR Green Supermix reagent (Bio-Rad Laboratories, Inc., Hercules, CA, USA) and 7.5 $\mu \mathrm{M}$ each of forward and reverse primers on an T100 thermal cycler (Bio-Rad Laboratories, Inc.). Primer sequences for the renal cortex samples were as follows: E-cadherin forward, 5'-GTC AAACGGCATCTAAAGC-3', and reverse, 5'-CAAAGA CCTCCTGGATAAACT-3'; $\alpha$-SMA, forward 5'-ACTGGG ACGACATGGAAAAG-3', and reverse, 5'-CATCTCCAG AGTCCAGCACA-3'; collagen I forward, 5'-GAGCGGAGA GTACTGGATCG-3', and reverse, 5'-TACTCGAACGGG AATCCATC-3'; and FN forward, 5'-CCATTGCAAATC GCTGCCAT-3', and reverse, 5'-AACATTTCTCAGCTA TTGGCTT-3'. In the NRK-52E cells, the primer sequences were as follows: E-cadherin forward, 5'-AACGAGGGC ATTCTGAAAACA-3', and reverse, 5'-CACTGTCACGTG CAGAATGTACTG-3'; $\alpha$-SMA forward, 5'-GACCCTGAA GTATCCGATAGAACA-3', and reverse, 5'-CACGCGAAG CTCGTTATAGAAG-3'; and FN forward, 5'-CATGGCTTT AGGCGAACCA-3', and reverse, 5'-CATCTACATTCGGCA GGTATGG-3' (Sangon Biotech Co., Ltd., Shanghai, China). In both cases, GAPDH was used as the reference gene and had the following primer sequences: Forward 5'-AGTGGC AAAGTGGAGATT-3' and reverse, 5'-GTGGAGTCATAC TGGAACA-3' (Sangon Biotech Co., Ltd.). The PCR cycling conditions were as follows: Predenaturation at $95^{\circ} \mathrm{C}$ for $10 \mathrm{~min}, 40$ cycles at $95^{\circ} \mathrm{C}$ for $15 \mathrm{sec}, 60^{\circ} \mathrm{C}$ for $1 \mathrm{~min}$ and $72^{\circ} \mathrm{C}$ for $20 \mathrm{sec}$, and a final extension at $60^{\circ} \mathrm{C}$ for $5 \mathrm{~min}$. The specificity of the qPCR was confirmed by $1 \%$ agarose gel electrophoresis and melting curve analysis. The relative mRNA expression levels were determined using the $2^{-\Delta \Delta \mathrm{Cq}}$ method (13). 


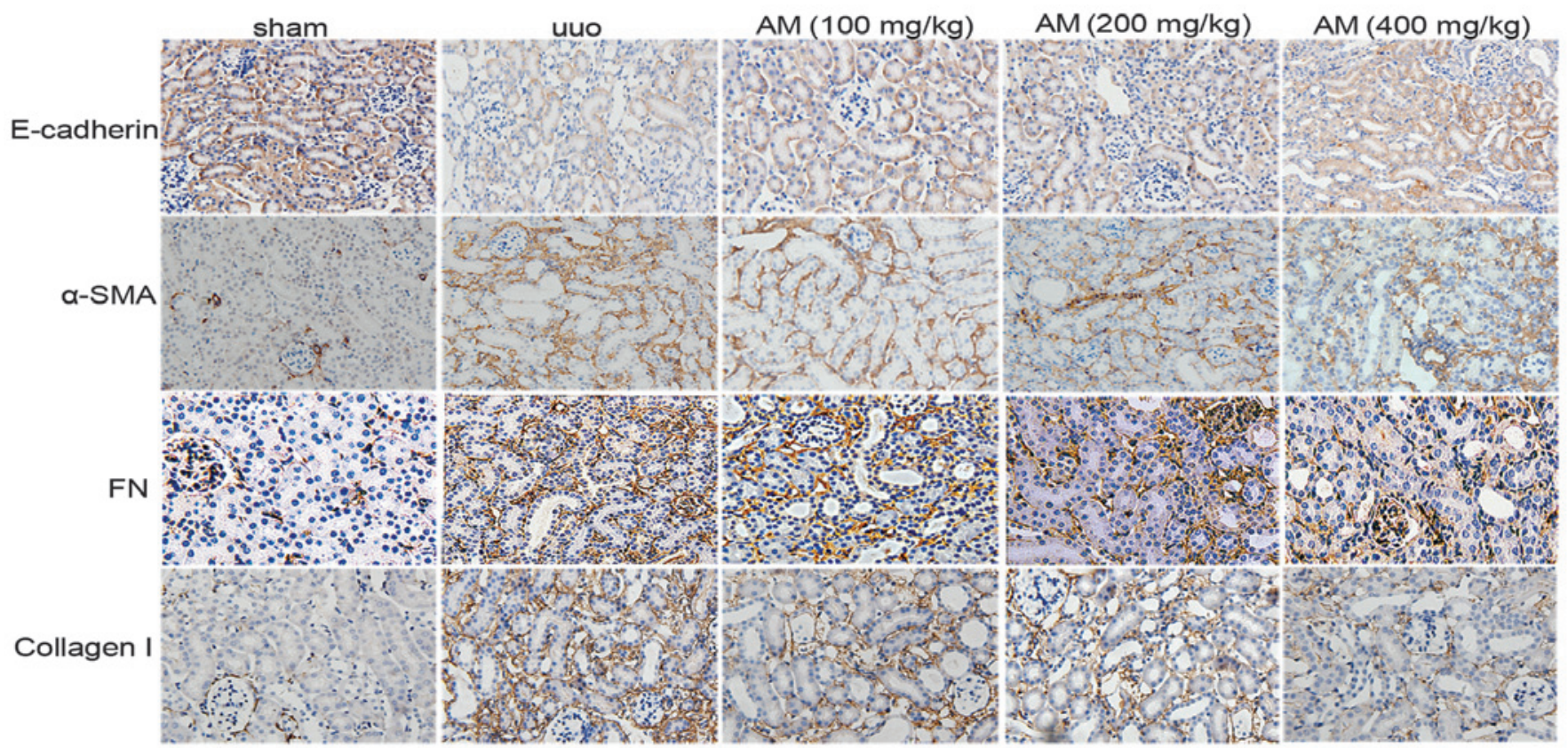

Figure 1. Renal paraffin sections were immunostained with antibodies targeting E-cadherin, $\alpha$-SMA, FN, and collagen I to determine epithelial-mesenchymal transition (magnification, x200). $\alpha$-SMA, FN, and collagen I were positively expressed in interstitial cells, whereas E-cadherin was detected in epithelial cells. In the sham group, no fibrotic septa or other lesions were detected in $\alpha$-SMA, FN, and collagen I-positive cells, whereas numerous stained epithelial cells were detected. In the UUO model group, numerous interstitial cell fibers were detected, and epithelial staining decreased. In the AM-treated group, $\alpha$-SMA, FN and collagen I expression decreased, whereas epithelial cells increased. SMA, smooth muscle actin; FN, fibronectin; UUO, unilateral ureteral obstruction; AM, Astragalus membranaceus.

A

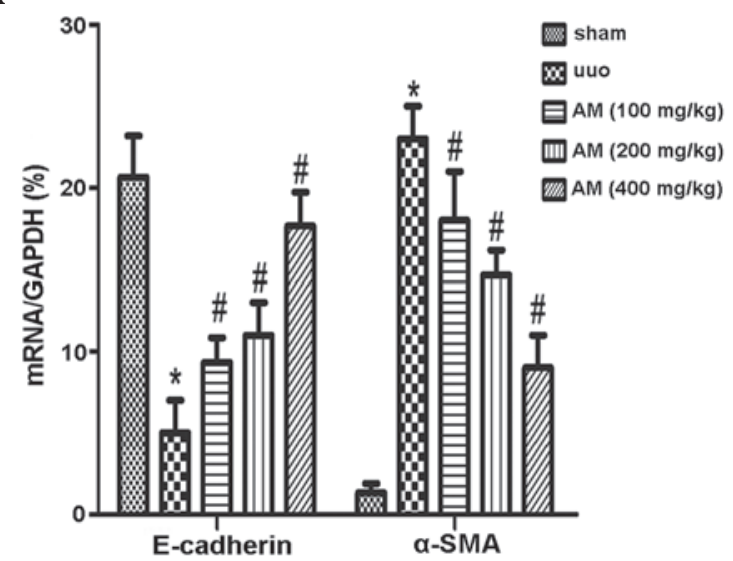

B

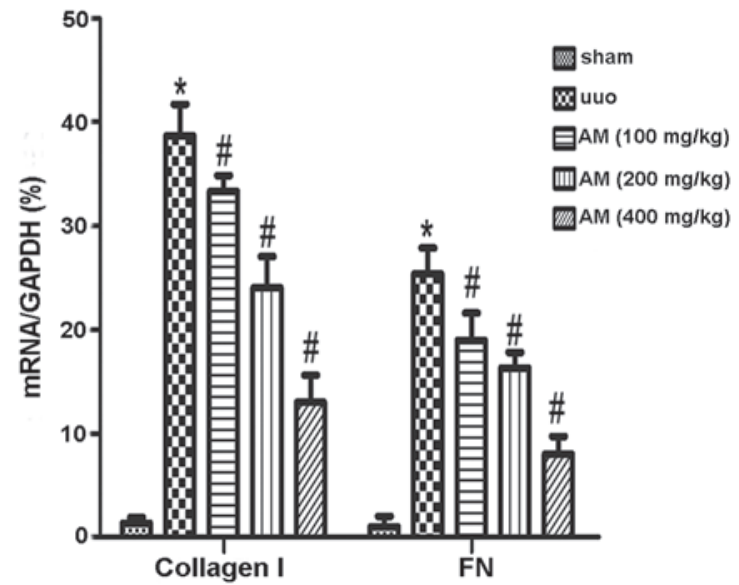

Figure 2. Analysis of the epithelial-mesenchymal transition in vitro. Reverse transcription-quantitative polymerase chain reaction was used to analyze the mRNA expression levels of (A) E-cadherin and $\alpha$-SMA, and (B) collagen I and FN in the sham, UUO, and UUO treated with AM (100, 200 or 400 mg/kg body weight) groups. Relative mRNA expression levels were quantified by densitometric analysis. Data are presented as the mean \pm standard deviation. ${ }^{*} \mathrm{P}<0.05$, vs. the sham group and ${ }^{\#} \mathrm{P}<0.05$, vs. the UUO group. SMA, smooth muscle actin; FN, fibronectin; UUO, unilateral ureteral obstruction; AM, Astragalus membranaceus.

Western blot analysis. Kidney and cell culture samples were resuspended in $0.4 \mathrm{ml}$ sodium dodecyl sulfate (SDS) lysis buffer containing $50 \mathrm{mM}$ Tris- $\mathrm{HCl}(\mathrm{pH} 7.4), 150 \mathrm{mM} \mathrm{NaCl}$, $0.1 \%$ SDS, $1 \%$ sodium deoxycholate, $1 \%$ Triton X-100, $1 \mathrm{mM}$ phenylmethylsulfonyl fluoride, $1 \mathrm{mM}$ EDTA and $5 \mu \mathrm{g} / \mathrm{ml}$ leupeptin (Beyotime Institute of Biotechnology). Protein concentration was determined using a BCA protein assay kit (cat. no. p0012s; Beyotime Institute of Biotechnology) and $30 \mu \mathrm{g}$ total protein/well was subsequently loaded and separated by $10 \%$ SDS-polyacrylamide gel electrophoresis. The gels were electroblotted onto polyvinylidene difluoride membranes (EMD Millipore, Billerica, MA, USA) and blocked with 5\% milk for $1 \mathrm{~h}$ prior to incubation with rabbit anti-Smad2/3 (1:1,000; sc-11769) and anti-TFG- $\beta 1$ $(1: 1,000 ;$ sc-146) polyclonal antibodies (both Santa Cruz Biotechnology, Inc., Dallas, TX, USA), and rabbit anti-GAPDH monoclonal antibody $(1: 1,000 ; 5174$; Cell Signaling Technology, Inc., Danvers, MA, USA), overnight at $4^{\circ} \mathrm{C}$. Following washing three times with Tris-buffered saline containing Tween-20, the membranes were incubated with HRP-conjugated goat anti-rabbit/mouse polyclonal secondary antibody $(1: 100$; G1201; Guge Biotechnology Co., Ltd.) for $1 \mathrm{~h}$ at room temperature. Bound antibodies were detected using an enhanced chemiluminescence 

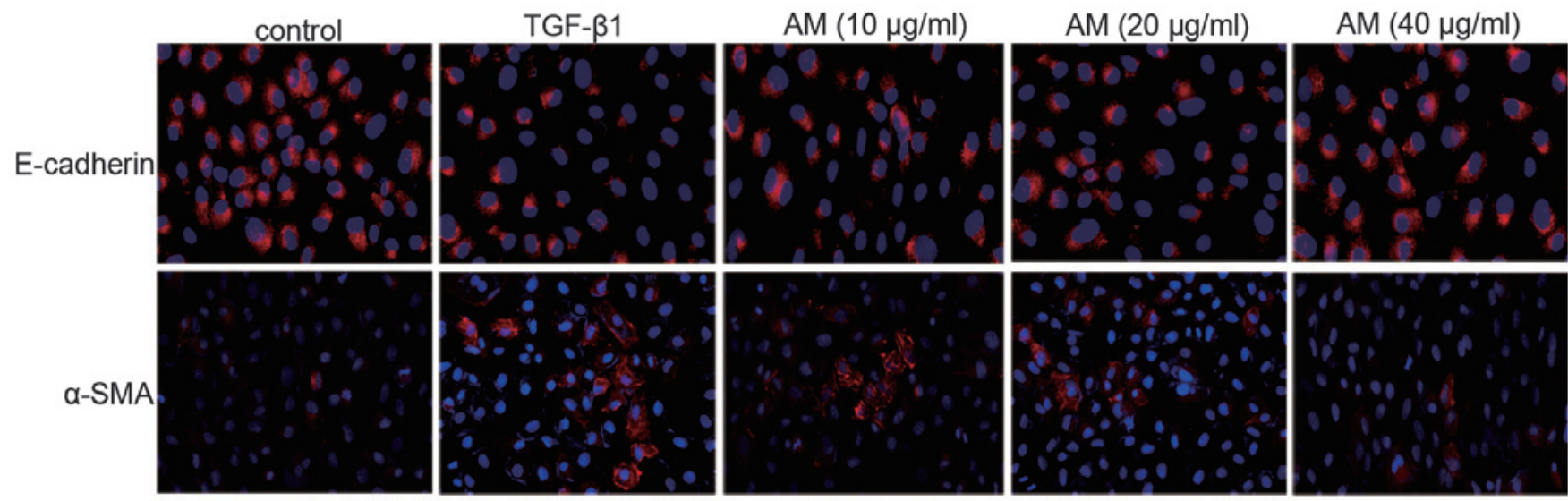

FN
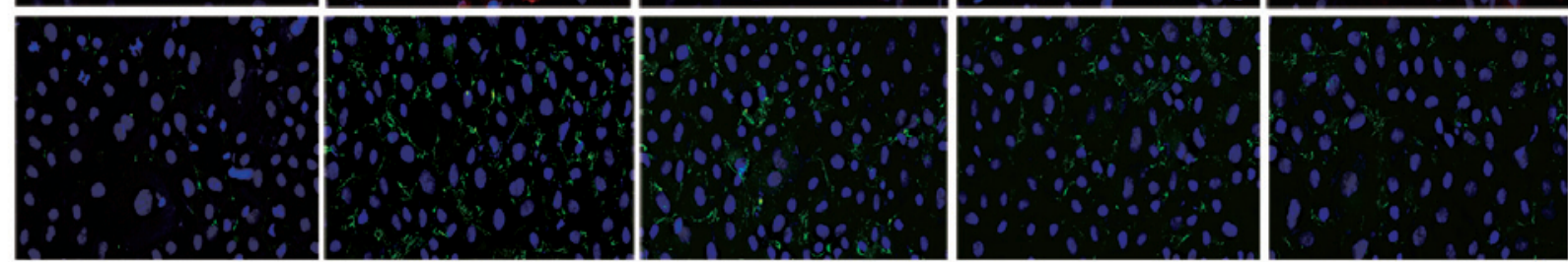

Figure 3. Murine renal proximal tubule NRK-52E cells were stained with E-cadherin, $\alpha$-SMA, and FN to determine epithelial-mesenchymal transition (magnification, x200). In the control group, no $\alpha$-SMA or FN-positive cells were detected, whereas numerous E-cadherin-positive cells were detected. E-cadherin expression decreased in the TGF- $\beta 1$ group, whereas the number of $\alpha$-SMA and FN-positive cells increased, as compared with the control group. Groups treated with 10,20, and $40 \mu \mathrm{g} / \mathrm{ml}$ AM exhibited reverse effects, as compared with the TGF- $\beta 1$ group. SMA, smooth muscle actin; FN, fibronectin; TGF, tumour growth factor; AM, Astragalus membranaceus.
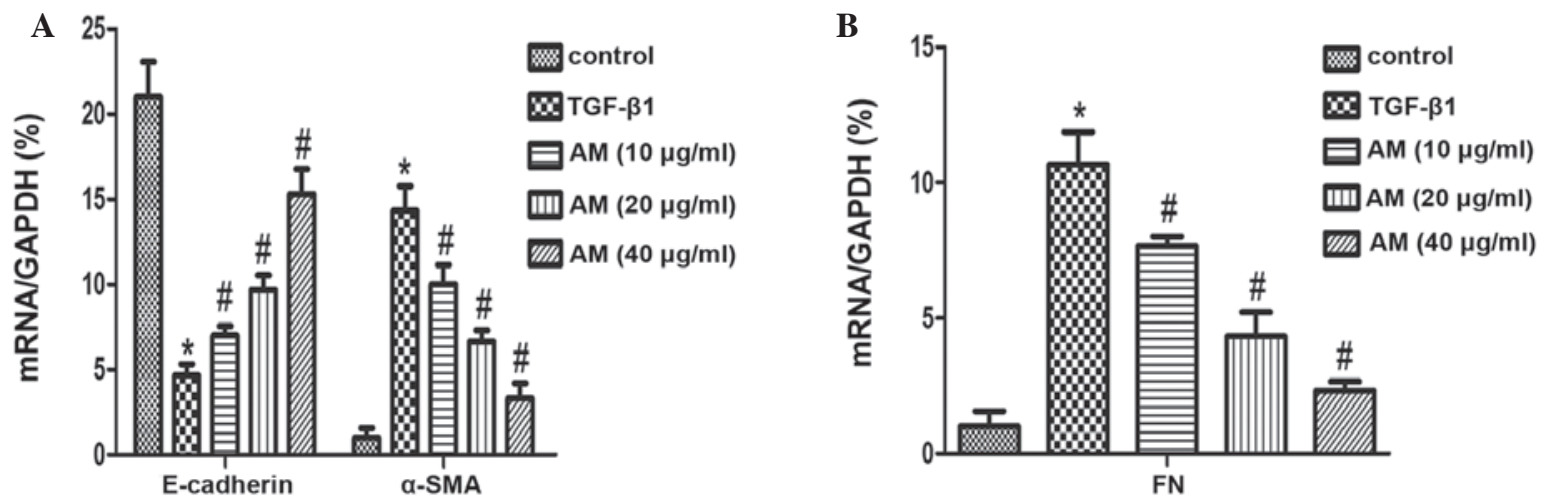

Figure 4. Analysis of the epithelial-mesenchymal transition in vivo. Reverse transcription-quantitative polymerase chain reaction was used to analyze the mRNA expression levels of (A) E-cadherin and $\alpha$-SMA, and (B) FN in the control, TGF- $\beta 1$, and TGF- $\beta 1$ treated with AM (10, 20 or $40 \mu \mathrm{g} / \mathrm{ml}) \mathrm{groups}$. Relative mRNA expression levels were quantified by densitometric analysis. Data are presented as the mean \pm standard deviation. ${ }^{*} \mathrm{P}<0.05$ vs. the control group; ${ }^{\#} \mathrm{P}<0.05$ vs. the TGF- $\beta 1$ group. SMA, smooth muscle actin; FN, fibronectin; TGF, tumour growth factor; AM, Astragalus membranaceus.

system (RPN998; GE Healthcare Life Sciences, Chalfont, UK). Band signals were detected using an Odyssey Infrared Imaging system Model 9120 (LI-COR Biotechnology, Lincoln, NE, USA), and band intensities were analyzed using Quantity-One software (Bio-Rad Laboratories, Inc.). Relative protein expression levels were normalized to GAPDH and compared with a control.

Statistical analysis. SPSS 17.0 software (SPSS, Inc., Chicago, IL, USA) was used for statistical analysis. Data were analyzed using one-way analysis of variance and Fisher's least significant difference t-test was used for multiple comparisons of two sample means. Data were presented as the mean \pm standard deviation. $\mathrm{P}<0.05$ was considered to indicate a statistically significant difference.

\section{Results}

AM upregulates $\alpha$-SMA and E-cadherin expression and decreases ECM in the kidneys of UUO mice. The effects of $\mathrm{AM}$ on the expression levels of $\alpha$-SMA, E-cadherin, and ECM were investigated during obstructive nephropathy, which is a well-characterized and widely-used model of renal interstitial fibrosis. Immunohistochemistry staining demonstrated that the protein expression of $\alpha$-SMA, collagen I, and FN in the renal interstitium were significantly elevated in the mouse kidney following obstructive injury, whereas E-cadherin protein expression decreased. AM markedly reversed the changes in the expression levels of the markers (Fig. 1).

The mRNA expression levels of $\alpha$-SMA significantly increased in the UUO group $(\mathrm{P}<0.05)$, whereas E-cadherin 


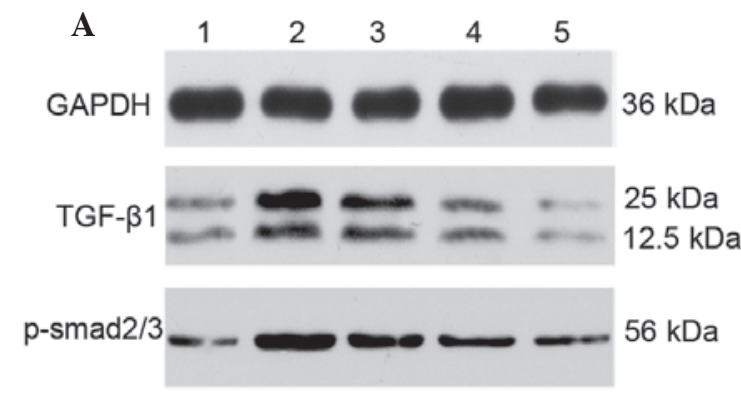

B

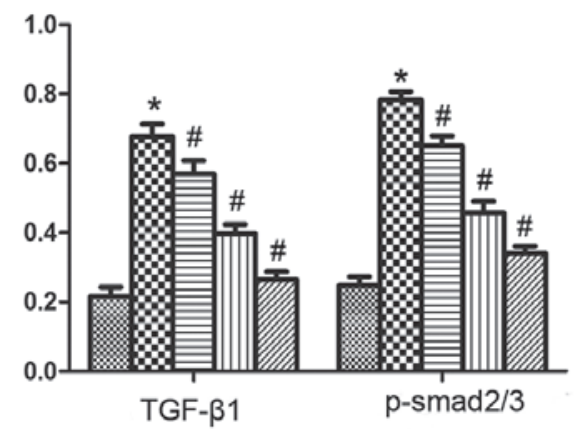

D

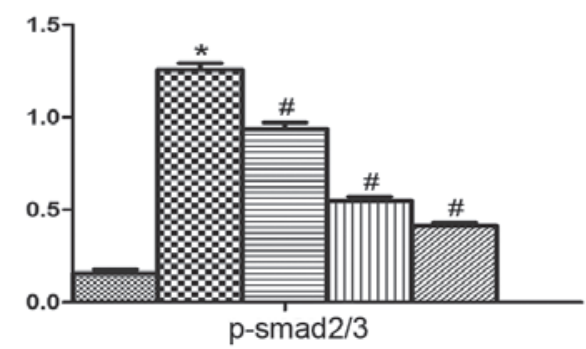

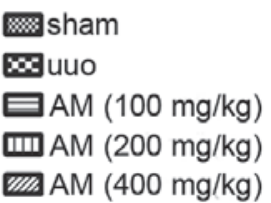

.

$\otimes$ TGF- $\beta 1$

曰AM $(10 \mu \mathrm{g} / \mathrm{ml})$

西AM $(20 \mu \mathrm{g} / \mathrm{ml})$

AM $(40 \mu \mathrm{g} / \mathrm{ml})$

Figure 5. Smad-dependent TGF- $\beta 1$ signaling in UUO mice and murine renal proximal tubule NRK-52E cells, as detected by western blotting and quantified using densitometric analysis. (A) Representative western blotting and (B) semiquantitative histograms of TGF- $\beta 1$ and p-Smad $2 / 3$ expression levels in UUO mice. Data are presented as the mean \pm standard deviation. ${ }^{*} \mathrm{P}<0.05$, vs. the sham group; ${ }^{~} \mathrm{P}<0.05$, vs. the UUO group. (C) Western blotting and (D) semiquantitative histograms of p-Smad2/3 expression in the groups treated with TGF- $\beta 1$ alone and TGF- $\beta 1$ with AM in NRK-52E cells. Data are presented as the mean \pm standard deviation. " $\mathrm{P}<0.05$, vs. the control group; ${ }^{\text {"P }}<0.05$, vs. the TGF- $\beta 1$ group. TGF, tumour growth factor; UUO, unilateral ureteral obstruction; $\mathrm{p}$ phosphorylated; AM, Astragalus membranaceus.

expression levels significantly decreased $(\mathrm{P}<0.05)$. As compared with the UUO group, downregulated $\alpha$-SMA, collagen I, and FN mRNA expression levels were detected in the AM groups, whereas E-cadherin mRNA expression levels were upregulated. The difference between the AM and UUO groups was determined to be significant $(\mathrm{P}<0.05)$, and the three AM treatment groups were significantly different from the UUO group ( $\mathrm{P}<0.05 ;$ Fig. 2). These data suggest that AM may be a potent agent for the reversal of renal tubular EMT in UUO mice.

AM upregulates $\alpha$-SMA and E-cadherin and decreases ECM in $N R K-52 E$ cells stimulated with TGF- $\beta 1$. Immunofluorescence analysis of the TGF- $\beta 1$-stimulated NRK-52E cells demonstrated that the protein expression of $\alpha$-SMA and FN increased, whereas E-cadherin protein expression decreased. AM administration markedly decreased $\alpha$-SMA protein expression and increased E-cadherin protein expression (Fig. 3), in a dose-dependent manner; therefore, the greatest AM concentration $(40 \mu \mathrm{g} / \mathrm{ml})$ markedly inhibited these changes. These results were concordant with the RT-qPCR analysis, which demonstrated reduced $\alpha$-SMA expression levels and increased E-cadherin expression levels following AM administration, as compared with the results following TGF- $\beta 1$ treatment without AM intervention ( $\mathrm{P}<0.05$; Fig. 4).

AM inhibits the TGF- $\beta 1$ signaling pathway and the phosphorylation of Smad2/3. Western blot analysis demonstrated that ureteral obstruction markedly increased the protein expression levels of TGF- $\beta 1$ and the phosphorylation of Smad2/3 in the UUO group, as compared with the sham group $(\mathrm{P}<0.05$; Fig. $5 \mathrm{~A}$ and $\mathrm{B})$. As compared with the UUO group, the AM treatment groups exhibited significantly reduced renal TGF- $\beta 1$ and phosphorylated Smad $2 / 3$ expression levels $(\mathrm{P}<0.05)$. Furthermore, western blot analysis demonstrated that Smad $2 / 3$ protein expression levels were significantly increased in the TGF- $\beta 1$-stimulated NRK-52E cells, as compared with the control group $(\mathrm{P}<0.05)$. Treatment with AM significantly downregulated $\mathrm{p}-\mathrm{Smad} 2 / 3$ protein expression levels (Fig. 5C and D). These data suggested that AM may effectively inhibit the expression and phosphorylation of the $\mathrm{Smad} 2 / 3$ protein in NRK-52E cells.

\section{Discussion}

The treatment efficacy of renal fibrosis remains unclear, however inhibition of EMT may serve as a novel therapeutic strategy to disrupt the process of fibrosis. The present study demonstrated that AM is capable of improving fibrosis by inhibiting expression level alterations associated with EMT in vivo and in vitro and investigating the underlying mechanisms, which may involve the TGF- $\beta 1 /$ Smad signaling pathway.

Renal interstitial fibrosis is a common pathological alteration, which is observed in various acute and chronic end-stage kidney diseases (14). The pathological basis of fibrosis is the production of ECM from myofibroblasts and it has been demonstrated that myofibroblasts predominantly originate through EMT (15). EMT is characterized by the loss of epithelial cells and the acquisition of mesenchymal markers due to excessive exposure to profibrotic cytokines (16). TGF- $\beta 1$ is a well-characterized fibrogenic cytokine which is associated with renal diseases and has a key role in EMT $(17,18)$. In the present study, TGF- $\beta 1$ was used to induce NRK-52E epithelial cell transformation into myoblasts. The results demonstrated that TGF- $\beta 1$ is capable of successfully inducing EMT, with decreased expression of the epithelial phenotype detected, via 
E-cadherin, accompanied by a dose-dependent increase in $\alpha$-SMA expression levels.

Due to the important role of EMT in renal fibrosis, any therapeutic strategy that targets the EMT may improve fibrosis (19). AM has been used in traditional Chinese medicine for thousands of years, where it is used to protect and support the immune system, prevent colds and upper respiratory infections, lower blood pressure, treat heart diseases and diabetes, and protect the liver (19). Previous studies have demonstrated the anti-fibrotic effects of AM by inhibiting the activation of the TGF- $\beta 1$ signaling pathway $(20,21)$. Furthermore, the potential anti-inflammatory effects of AM have been demonstrated in various in vitro studies $(22,23)$. Notably, previous studies demonstrated that AM was beneficial in reducing renal tubulointerstitial fibrosis $(24,25)$; however, the underlying pharmacological mechanisms have yet to be elucidated.

The present study demonstrated that treatment with AM may improve TGF- $\beta 1$-induced renal fibrosis, and Smad2/3 signaling pathway activation may be an important factor in this process, since TGF- $\beta 1$ signaling pathways are activated by receptor phosphorylation prior to subsequent phosphorylation. In particular, R-Smad proteins are phosphorylated by TGF- $\beta$ type I receptors, leading to the activation of a series of downstream events $(26,27)$. The results of the present in vitro experiment demonstrated that TGF- $\beta 1$ stimulation of NRK-52E cells increased the expression levels of the Smad2/3 protein, which were subsequently reduced by co-treatment with AM. This suggested that AM inhibits the TGF- $\beta 1 / \mathrm{Smad}$ signaling pathway via the phosphorylation of Smad proteins, which have an important role in the activation of the TGF- $\beta 1$ signaling pathway.

In conclusion, the results of the present study suggested that AM may inhibit renal fibrosis; as indicated by the antagonized tubular EMT and ECM accumulation via TGF- $\beta / \mathrm{Smad} 2 / 3$. Therefore, AM may be a therapeutic agent for the prevention of renal fibrosis.

\section{References}

1. Liu Y: Cellular and molecular mechanisms of renal fibrosis. Nat Rev Nephrol 18: 684-696, 2011.

2. Liu Y: Renal fibrosis: New insights into the pathogenesis and therapeutics. Kidney Int 69: 213-217, 2006.

3. Falke LL, Gholizadeh S, Goldschmeding R, Kok RJ and Nguyen TQ: Diverse origins of the myofibroblast - implications for kidney fibrosis. Nat Rev Nephrol 11: 233-244, 2015.

4. Liu Y: New insights into epithelial-mesenchymal transition in kidney fibrosis. J Am Soc Nephrol 21: 212-222, 2010.

5. Zeisberg M and Duffield JS: Resolved: EMT produces fibroblasts in the kidney. J Am Soc Nephrol 21: 1247-1253, 2010.

6. Zavadil J, Cermak L, Nieves NS and Böttinger EP: Integration of TGF- $\beta /$ Smad and Jagged $1 /$ Notch signalling in epithelial-to-mesenchymal transition. EMBO 10 23: 1155-1165, 2004.

7. López-Hernández FJ and López-Novoa JM: Role of TGF- $\beta$ in chronic kidney disease: An integration of tubular, glomerular and vascular effects. Cell Tissue Res 347: 141-154, 2012.

8. Na D, Liu FN, Miao ZF, Du ZM and Xu HM: Astragalus extract inhibits destruction of gastric cancer cells to mesothelial cells by anti-apoptosis. World J Gastroenterol 15: $570-577,2009$.
9. Yuan C, Pan X, Gong Y, Xia A, Wu G, Tang J and Han X: Effects of Astragalus polysaccharides (APS) on the expression of immune response genes in head kidney, gill and spleen of the common carp, Cyprinus carpio L. Int Immunopharmacol 8: 51-58, 2008.

10. Gao J, Feng LJ, Huang Y, Li P, Xu DJ, Li J and Wu Q: Total glucosides of Danggui Buxue Tang attenuates bleomycin-induced pulmonary fibrosis via inhibition of extracellular matrix remodelling. J Pharm Pharmacol 64: 811-820, 2012.

11. Du JX, Sun MY, Du GL, Li FH, Liu C, Mu YP, Chen GF, Long AH, Bian YQ, Liu J, et al: Ingredients of Huangqi decoction slow biliary fibrosis progression by inhibiting the activation of the transforming growth factor-beta signaling pathway. BMC Complement Altern Med 12: 33, 2012.

12. Li Z, Zhang L, He W, Zhu C, Yang J and Sheng M: Astragalus membranaceus inhibits peritoneal fibrosis via monocyte chemoattractant protein (MCP)-1 and the transforming growth factor- $\beta 1$ (TGF- $\beta 1$ ) pathway in rats submitted to peritoneal dialysis. Int J Mol Sci 22: 12959-12971, 2014.

13. Livak KJ and Schmittgen TD: Analysis of relative gene expression data using real-time quantitative PCR and the 2(-Delta Delta C(T)) Method. Methods 25: 402-408, 2001.

14. Yang J and Liu Y: Dissection of key events in tubular epithelial to myofibroblast transition and its implications in renal interstitial fibrosis. Am J Pathol 159: 1465-1475, 2001.

15. Lan HY: Tubular epithelial-myofibroblast transdifferentiation mechanisms in proximal tubule cells. Curr Opin Nephrol Hypertens 12: 25-29, 2003.

16. Flier SN, Tanjore H, Kokkotou EG, Sugimoto H, Zeisberg M and Kalluri R: Identification of epithelial to mesenchymal transition as a novel source of fibroblasts in intestinal fibrosis. J Biol Chem 285: 20202-20212, 2010.

17. Matsuda H, Fukuda N, Ueno T, Katakawa M, Wang X, Watanabe T, Matsui S, Aoyama T, Saito K, Bando T, et al: Transcriptional inhibition of progressive renal disease by gene silencing pyrrole-imidazole polyamide targeting of the transforming growth factor- $\beta 1$ promoter. Kidney Int 79: 46-56, 2011.

18. Hills CE and Squires PE: The role of TGF- $\beta$ and epithelial-to mesenchymal transition in diabetic nephropathy. Cytokine Growth Factor Rev 22: 131-139, 2011.

19. Sun WY, Wang L, Liu H, Li X and Wei W: A standardized extract from Paeonia lactiflora and Astragalus membranaceus attenuates liver fibrosis induced by porcine serum in rats. Int J Mol Med 29: 491-498, 2012.

20. Yang Y, Yang S, Chen M, Zhang X, Zou Y and Zhang X: Compound Astragalus and Salvia miltiorrhiza extract exerts anti-fibrosis by mediating TGF-beta/Smad signaling in myofibroblasts. J Ethnopharmacol 118: 264-270, 2008.

21. Chen B, Li R, Yan N, Chen G, Qian W, Jiang HL, Ji C and Bi ZG: Astragaloside IV controls collagen reduction in photoaging skin by improving transforming growth factor- $\beta /$ Smad signaling suppression and inhibiting matrix metalloproteinase-1. Mol Med Rep 11: 3344-3348, 2015.

22. Huang WM, Liang YQ, Tang LJ, Ding Y and Wang XH: Antioxidant and anti-inflammatory effects of Astragalus polysaccharide on EA.hy926 cells. Exp Ther Med 6: 199-203, 2013.

23. Lai PK, Chan JY, Wu SB, Cheng L, Ho GK, Lau CP, Kennelly EJ, Leung PC, Fung KP and Lau CB: Anti-inflammatory activities of an active fraction isolated from the root of Astragalus membranaceus in RAW 264.7 macrophages. Phytother Res 28: 395-404, 2014.

24. Che X, Wang Q, Xie Y, Xu W, Shao X, Mou S and Ni Z: Astragaloside IV suppresses transforming growth factor- $\beta 1$ induced fibrosis of cultured mouse renal fibroblasts via inhibition of the MAPK and NF- $\kappa$ B signaling pathways. Biochem Biophys Res Commun 464: 1260-1266, 2015.

25. Zuo C, Xie XS, Qiu HY, Deng Y, Zhu D and Fan JM: Astragalus mongholicus ameliorates renal fibrosis by modulating HGF and TGF-beta in rats with unilateral ureteral obstruction. J Zhejiang Univ Sci B 10: 380-390, 2009.

26. Böttinger EP and Bitzer M: TGF-beta signaling in renal disease. J Am Soc Nephrol 13: 2600-2610, 2002.

27. Verrecchia F and Mauviel A: Transforming growth factor-beta signaling through the Smad pathway: Role in extracellular matrix gene expression and regulation. J Invest Dermatol 118: 211-215, 2002. 